\title{
Scientific support for innovative development of wheat bread long-term storage production
}

\author{
Natalia Zavorokhina*, Natalia Pankratyeva, and Nadezhda Goncharova \\ Ural State University of Economics, 620144 Yekaterinburg, Russia
}

\begin{abstract}
Nowadays in the context of innovative development the bread intended for long-term storage is necessary in hard-to-reach areas of Sverdlovsk region, participants of expeditions, workers of logging, geological parties, etc. Taking into account the constant demand for such products, it is necessary to develop new approaches to their creation. The aim of the study was the development of long-term storage bread from wheat flour, preserving consumer characteristics throughout the shelf life.The synergism of betulin,nisin and ascorbic acid was studied for the first time when they are used together to inhibit potato bread disease (hereinafter $\mathrm{CBH}$ ). It is proved that the use of additives, including $0.015 \%$ nisin together with $0.005 \%$ betulin to the mass of flour increases the shelf life of wheat bread up to 7 days since betulin inhibits $\mathrm{CBH}$, nisin inhibits the development of mold under $\mathrm{pH}$ conditions in the range of 5.5-6.5.For the first time, a technique is proposed for producing betulin nanosuspension with a high degree of dispersion. The effect of betulin nanosuspension with emulsifiers of calcium lactylate, polyglycerol fatty acid esters, lecithin on the inhibition of $\mathrm{CBH}$ and an increase in the shelf life of wheat bread was studied.
\end{abstract}

\section{Introduction}

Development of recipes of wheat bread, preserving nutritional value and a set of necessary consumer properties within 7-10 days is one of the innovative directions of the food industry. In the context of a digital economy the development of this direction is primarily due to the territorial length of the Russian Federation and the complexity of logistics operations in severe climatic conditions. In the strategy for improving the quality of food products in the Russian Federation until 2030, it was noted that "the consumption of food products with low consumer properties is the reason for reducing the quality of life and the development of a number of diseases of the population, including due to unreasonably high calorie content of food products, reduced nutritional value, excess saturated fat intake, micronutrient and fiber deficiency". In recent years, there has been a steady tendency in Russia to reduce the production of non-durable bread [1-18].

\section{Body of paper}

\footnotetext{
${ }^{*}$ Corresponding author: degustator@olympus.ru
} 
Production volume decreases on average by $2 \%$ per year. By the end of 2019, 5957 thousand tons of non-food baking were produced, i.e. 859 thousand tons less than in 2010 [19].

At the same time, the production of bakery products for long-term storage and baking of bakery products with low humidity is growing, as well as the production of bakery products during the same period. In 2019, 468.1 thousand tons of such products were produced, i.e. almost 30\% more than in 2010 - 363 thousand tons [20].

Sverdlovsk region is one of the most promising regions of the Russian Federation with a high level of commercial, cultural and social activity. The region demonstrates strong and stable economic growth and is among the top ten regions of the Russian Federation for most of the main indicators of socio-economic development. The production of long-term bread and confectionery in the Sverdlovsk region is the basis for the formation of the agrifood market and ensures food security in region. These products fully meet the internal needs of the region in terms of production and the range of products while processing domestic raw materials.

The development of wheat bread with a long shelf life will increase the supply of bread, reducing the cost of daily transportation.

The properties of bread change continuously, starting from the end of baking. The evaporation of moisture from baked bread leads to a decrease in its mass.

The bread is stale, which is reflected in the change in the properties of the crumb, which hardens and crumbles easily; cracks form in the crumb.

Along with drying and staling during long-term storage, especially in adverse conditions, bread molds and is spoiled. All changes that occur in bread, reduce its nutritional value. As a result, it is considered to be 2-3 days as a normal shelf life of bread.

In order to deliver bread to hard-to-reach areas during emergency situations, it becomes necessary to provide bread for 10-12 days. During this time, bread should not be moldy, quick callous and should retain its inherent taste and aroma.

The development of long-term storage bread from wheat flour is timely and relevant. There is an increasing demand of retail chains for this assortment of bread.

To solve this problem, in the course of scientific research, the effectiveness of the methods that extend the shelf life of bread from wheat flour was substantiated.

The defining criteria were: inhibition of potato bread disease, organoleptic indicators.

1. When developing a formulation with a complex addition of nisin + betulin + ascorbic acid, we took into account the influence of the components on the baking properties of wheat flour, the quantity and quality of gluten, on inhibition of mold and Bacillus subtilis, organoleptic and physico-chemical indicators of the quality of finished products.

Studies of the qualitative characteristics of the dough and bread using a complex additive showed that the additive does not adversely affect the speed of biochemical processes, does not slow down the process of gas formation during the fermentation of the dough [21]. Ascorbic acid in the composition of the additive stabilizes the process, slightly strengthening the fraction of gluten proteins.

Physico-chemical indicators of the quality of bread with the additive did not significantly differ from the control sample [22]; porosity and humidity of the finished products were approximately at the same level, which corresponded to the requirements of regulatory documents for bread made from wheat flour. The specific volume of bread was at the level of $3.4 \mathrm{~cm} / \mathrm{g} 3$, the form stability of the hearth bread was 0.45 .

The introduction of a complex additive nisin + betulin + ascorbic acid in the bread recipe from wheat flour allowed to obtain a product with an extended shelf life of 7 days.

The antimicrobial effect of the components of the additive - nisin and betulin helps to prevent the formation of mold and the manifestation of signs of disease of potato bread disease throughout the term shelf life of bread. There was a persistent effect of inhibition of 
Bacillus subtilis with the introduction of additives throughout the shelf life of 10 days. The number of Bacillus subtilis colonies decreased in proportion to the increase in the active components in the additive - nisin and betulin. The recommended optimal dosage of a complex additive in the dough (option 2), which corresponds to the mass fraction of nisin: betulin: ascorbic acid, is $0.005: 0.015: 0.003 \%$ by weight of flour, respectively. It is not advisable to increase the dosage of these substances, since the goal of inhibiting Bacillus subtilis has been achieved.

However, the structural and mechanical characteristics of the crumb with a complex addition of nisins + betulin + ascorbic acid, during storage of bread, shifted towards elastic deformation. The process of stale bread with the addition of additives went less intensively, but after 7 days of storage of bread there were significant changes in the structure of the crumb, the bread was rated as "moderately stale" and "stale", which corresponds to a total of $58.2-41.0$ and 37 points , $4-37.0$, respectively. A control sample of bread on the 7th day of storage was evaluated as "stale" - 38.0 points, on the 10th day of storage - "very stale" -18 points.

2. For the purpose of scientific research of the staling process, we studied a way to preserve the freshness of bread from flour with a low amylose content.

When storing baked bread in its crumb, starch is retrograded, i.e. partial reverse transition of starch to a crystalline state, approaching that in which the starch was in the test before baking. At the same time, the structure of starch is compacted, its solubility decreases and partial absorption of moisture absorbed during gelatinization occurs.

Starch granules are composed of two types of molecules - amylose and amylopectin. In wheat, the amylose content ranges from 20-25\%, amylopectin - 75-80\%. Amylopectin retrograde slower and to a lesser extent than amylose.

Wheat flour bread was compared with the highest amylose content of $28 \%$ (Tsar, manufacturer of Union Soyuzpishcheprom LLC) and the lowest amylose content of $13 \%$ (Molino Grassi, Italy).

According to the organoleptic indicators, no significant differences between the bread samples were observed. The porosity of bread from flour with a low amylose content was $5 \%$ higher, the volumetric yield of bread was $224 \mathrm{~cm} 3 / 100 \mathrm{~g}$ against the volumetric yield of the control sample $-218 \mathrm{~cm} 3 / 100$.

During the storage of the experimental samples, the staling process was restrained; the indicators forming consumer qualities of bread from flour with a low amylose content were higher than the control sample.

Thus, the value of the strain index of bread crumb from Molino Grassi flour is $20 \%$ higher than the control sample. The moisture content of bread from flour with a low amylose content for 10 days of storage is higher than the control sample by $2 \%$. Such bread retains moisture longer, respectively, the hydrophilicity index of the crumb, shrinkage is higher than that of the sample, with the usual content of amylose.

Evaluation of the freshness of bread on a scale showed that bread with a low amylose content corresponded to the category of "fresh bread" for 7 days, and the control sample corresponded to the category of "stale".

Flour with a low amylose content does not have a positive effect on the inhibition of mold and Bacillus subtilis, this aspect was not the aim of the study.

3. The use of betulin in the form of nanosuspension with the emulsifier lecithin is the most effective way to preserve the consumer characteristics of bread during 10 days of storage.

The antimicrobial effect of betulin in the emulsion is enhanced by its uniform distribution over the mass of the dough. The emulsifier lecithin maintains the stability of the emulsion and promotes an even distribution of all components. 
The technological scheme for obtaining microsuspension by the condensation method is shown in Figure 1:
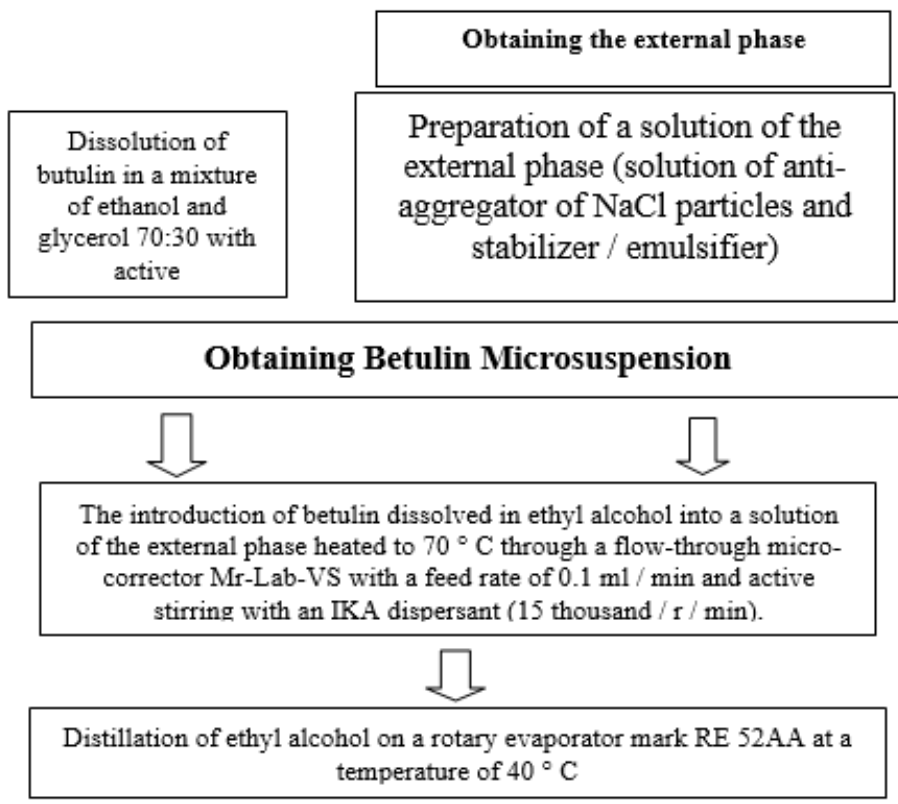

Fig. 1.Technological scheme for producing a suspension of betulin by the condensation method.

Table 1 shows the rheological, physico-chemical and organoleptic characteristics of the best bread samples from wheat flour grade 1 with nanosuspension of betulin.

Table 1.The results of studies of samples of HPM with nanosuspension of betulin, $n=3$.

\begin{tabular}{|c|c|c|c|c|}
\hline \multirow[b]{2}{*}{ Indicator } & \multicolumn{4}{|c|}{ Potato bread disease } \\
\hline & The control & $\begin{array}{c}\text { Sample No. } \\
1\end{array}$ & $\begin{array}{l}\text { Sample No. } \\
2\end{array}$ & $\begin{array}{c}\text { Sample No. } \\
3\end{array}$ \\
\hline \multicolumn{5}{|c|}{ Physical and chemical indicators } \\
\hline $\begin{array}{l}\text { The porosity of } \\
\text { the crumb, } \%\end{array}$ & $73,0 \pm 1,0$ & $76,0 \pm 1,0$ & $74,0 \pm 1,0$ & $77,8 \pm 1,0$ \\
\hline $\begin{array}{l}\text { Specific volume, } \\
\mathrm{cm} 3 / \mathrm{g} / / \mathrm{g}\end{array}$ & $3,40 \pm 0,2$ & $3,85 \pm 0,2$ & $3,62 \pm 0,2$ & $4,40 \pm 0,2$ \\
\hline $\begin{array}{l}\text { Shape stability } \\
\text { (H/D) }\end{array}$ & $0,45 \pm 0,1$ & $0,48 \pm 0,1$ & $0,46 \pm 0,1$ & $0,52 \pm 0,1$ \\
\hline $\begin{array}{l}\text { Humidity of the } \\
\text { crumb, } \%\end{array}$ & $41,0 \pm 0,5$ & $42,0 \pm 0,5$ & $42,0 \pm 0,5$ & $42,5 \pm 0,5$ \\
\hline Acidity, degrees & $2,5 \pm 0,2$ & $2,5 \pm 0,2$ & $2,2 \pm 0,2$ & $2,6 \pm 0,2$ \\
\hline \multicolumn{5}{|c|}{ Rheological indicators } \\
\hline $\begin{array}{l}\text { Structural and } \\
\text { mechanical } \\
\text { properties of the } \\
\text { crumb, } \\
\qquad \mathrm{H} \text { total }\end{array}$ & $17,2 \pm 1,5$ & $18,6 \pm 1,8$ & $19,8 \pm 2,0$ & $21,4 \pm 1,7$ \\
\hline$\Delta$ Hplastic & $12,2 \pm 0,1$ & $12,8 \pm 0,5$ & $13,6 \pm 0,6$ & $14,2 \pm 0,2$ \\
\hline$\Delta \mathrm{H}$ elastic & $5,0 \pm 0,2$ & $5,8 \pm 0,5$ & $6,2 \pm 0,22$ & $7,2 \pm 0,4$ \\
\hline
\end{tabular}




\begin{tabular}{|c|c|c|c|c|}
\hline Appearance & \multicolumn{4}{|c|}{ Appropriate bread form. Rectangular, right } \\
\hline surface & \multicolumn{4}{|c|}{ Smooth, no cracks or blasts } \\
\hline colour & \multicolumn{4}{|c|}{ Light brown, uniform } \\
\hline $\begin{array}{c}\text { Crumb condition, } \\
\text { baking }\end{array}$ & \multicolumn{4}{|c|}{ Baked } \\
\hline knead & \multicolumn{4}{|c|}{ No trace of impurity } \\
\hline Porosity & $\begin{array}{l}\text { Sufficiently } \\
\text { developed } \\
\text { and } \\
\text { uniform, } \\
\text { pores small } \\
\text { and } \\
\text { medium, } \\
\text { thin-walled }\end{array}$ & $\begin{array}{l}\text { Sufficiently } \\
\text { developed } \\
\text { and } \\
\text { uniform, } \\
\text { pores small } \\
\text { and } \\
\text { medium, } \\
\text { thin-walled }\end{array}$ & $\begin{array}{l}\text { Well } \\
\text { developed, } \\
\text { uniform, } \\
\text { pores small } \\
\text { and thin- } \\
\text { walled }\end{array}$ & $\begin{array}{c}\text { Well } \\
\text { developed, } \\
\text { uniform, } \\
\text { pores small } \\
\text { and thin- } \\
\text { walled }\end{array}$ \\
\hline Taste & \multicolumn{4}{|c|}{ Pronounced, characteristic for wheat bread } \\
\hline Smell & \multicolumn{4}{|c|}{ Pronounced, characteristic for wheat bread } \\
\hline
\end{tabular}

\section{Conclusion}

As a result of the experiment, data were obtained confirming the extension of the shelf life of bread to 10 days.

It was found that a bread sample with betulin nanosuspension and lecithin corresponded to the "fresh" category until the expiration date of 10 days. No evidence of mold or Bacillus subtilis was observed.

Considering the above, the priority method of extending the shelf life of bread from wheat flour can be considered a method using betulin in the form of nanosuspension in combination with an emulsifier lecithin.

\section{References}

1. Guo Chen,Camilla Öhgren,Maud Langton,Kaare F. Lustrup, Jan Swenson, J.of Cereal Science, 57 (1), 120 (2013)

2. Yao Zhang, Dandan Li, Na Yang,ZhengyuJin, Xueming Xu, LWT, 98, 39 (2018)

3. Yao Zhang, LunanGuo, Dan Xu, Dandan Li, Xueming Xu, Food Chemistry, 256 (1), 373 (2018)

4. Denglin Luo, Ruoyan Wu, Jie Zhang, Kangyi Zhang, Xuan Li, J. of Cereal Science, (83), 147 (2018)

5. Fabio Licciardello, Virgilio Giannone, Matteo Alessandro Del Nobile, GiueppMuratore, Antonella Pasqualone, Food Chemistry, 224, 181 (2017)

6. Johannes Frauenlob, Maria ElettaMoriano, Ute Innerkofler, Stefano D'Amico, Regine Schoenlechner, J. of Cereal Science, 77, 58 (2017)

7. Elena Mellado-Ortega, DámasoHornero-Méndez, Food Research Int., 99 (2), 877 (2017) 
8. Seong-Woo Cho, Chon-Sik Kang, HyeonSeokKo, Byung-KeeBaik, Chul Soo Park, J. of Integrative Agriculture, 17 (8), 1706 (2018)

9. Michael G. Gänzle, Jinshui Zheng, Int. J. of Food Microbiology, (to be published)

10. Carlo Giuseppe Rizzello, Michela Verni, Stefano Bordignon, Valerio Graaglia, Marco Gobbetti, Food Microbiology, 64, 72 (2017)

11. Lauren Tebben, Yanting Shen, Yonghui Li, Trends in Food Science \& Technology, 81, 10 (2018)

12. Yao Zhang, Dandan Li, Na Yang, ZhengyuJin, Xueming Xu, LWT, 98, 39 (2018)

13. HabtuShumoy, Filip Van Bockstaele, Dilara Devecioglu, Katleen Raes, Food Chemistry, 264, 34-40 (2018)

14. Yao Zhang, LunanGuo, Dan Xu, Dandan Li, Xueming Xu, Food Chemistry 256, 373 (2018)

15. Fabio Licciardello, Virgilio Giannone, Matteo Alessandro Del Nobile, Giuseppe Muratore, Antonella Pasqualone, Food Chemistry, 224, 181 (2017)

16. Johannes Frauenlob, Maria ElettaMoriano, Ute Innerkofler, Stefano D'Amico, Regine Schoenlechner, J.of Cereal Science, 77, 58 (2017)

17. Elena Mellado-Ortega, DámasoHornero-Méndez, Food Research International, 99 (2), 877 (2017)

18. Seong-Woo Cho, Chon-Sik Kang, HyeonSeokKo, Byung-KeeBaik, Chul Soo Park, J. of Integrative Agriculture, 17 (8), 1706 (2018)

19. Natalia Zavorohina, Natalia Pankratieva, Nadezhda Goncharova, Advances in Social Science, Education and Humanities Research, 240, 62 (2019)

20. Natalia V. Zavorohina, Natalia A. Pankratieva, Nadezhda A. Goncharova, Improving preservation methods consumer characteristics of bread long-term storage, International Scientific Conference, Fifth Technological Order: Prospects for the Development and Modernization of the Russian Agro-Industrial Complex, (2019)

21. Denglin Luo, Ruoyan Wu, Jie Zhang, Kangyi Zhang, Xuan Li, J. of Cereal Science, 83, 147 (2018)

22. C.J. Lee, J.H. Seok, G.M. Hur, al, Planta Med, 70 (12), 1119 (2004) 